\title{
Research Needs and Strategies to Establish Best Practices and Cost Effective Models for Chronic Critical Illness
}

\author{
Shannon S Carson MD
}

\author{
Introduction \\ Prevention of Chronic Critical Illness \\ Patient Management \\ Communication and End-of-Life Care \\ Care Settings \\ Summary
}

\begin{abstract}
Past research in chronic critical illness has been effective in defining the population and identifying unique aspects of their outcomes and resource needs, but there has been little research focused on interventions to improve outcomes. This review discusses some research priorities that could have immediate impact on patient outcomes. General topics include prevention or limiting the incidence of chronic critical illness; specific topics related to patient management, such as interventions for weaning, rehabilitation, nutrition or infections; and methods to enhance communication and endof-life care. In addition to specific patient management interventions, further comparative effectiveness research on care settings is indicated, considering the expected growth in the patient population and the substantial resource needs. Key words: chronic critical illness; mechanical ventilation; outcomes. [Respir Care 2012;57(6):1014-1018. (C) 2012 Daedalus Enterprises]
\end{abstract}

\section{Introduction}

Research opportunities in the field of chronic critical illness (CCI) are numerous. Most of the research to date

Shannon S Carson MD is affiliated with the Division of Pulmonary and Critical Care Medicine, University of North Carolina School of Medicine, Chapel Hill, North Carolina.

Dr Carson presented a version of this paper at the 49th RESPIRATORY CARE Journal Conference, "The Chronically Critically Ill Patient," held September 9-10, 2011, in St Petersburg, Florida.

Dr Carson was partly supported by grants from the National Heart, Lung, and Blood Institute and the National Institute of Nursing Research, and has disclosed a relationship with RTI International.

Correspondence: Shannon S Carson MD, Division of Pulmonary and Critical Care Medicine, University of North Carolina School of Medicine, 4125 Bioinformatics Building, CB\#7020, Chapel Hill NC 27599. E-mail: scarson@med.unc.edu.

DOI: $10.4187 /$ respcare.01910 has focused on epidemiology, outcomes, and costs. This has been effective in defining the population and some of their problems, which is the first step in furthering research interest. But little has been accomplished in designing and studying interventions to improve patient outcomes or effectively reduce costs of care. This paper will review some research topics that should be of high priority for investigators and funding agencies. As in any field of medical research, the number of questions can be large, and it will not be possible to consider all of them. But some immediate issues will be reviewed in areas of prevention through dissemination of evidence-based practices, patient management, communication and end-of-life care, and care settings (Table 1).

\section{Prevention of Chronic Critical Illness}

Prevention of CCI should be a priority of every ICU, and a number of management principles have been proven efficacious for reducing duration of ventilation and the 
Table 1. Research Needs in Chronic Critical Illness

\begin{tabular}{|c|c|}
\hline General Topic & Research Priorities \\
\hline $\begin{array}{l}\text { Prevention of chronic } \\
\text { critical illness }\end{array}$ & $\begin{array}{l}\text { Effective dissemination and implementation } \\
\quad \text { of evidence-based practices } \\
\text { Early versus late tracheostomy } \\
\text { Prevention and treatment of delirium } \\
\text { Early ICU mobility }\end{array}$ \\
\hline $\begin{array}{l}\text { Management of the } \\
\text { chronically critically } \\
\text { ill patient }\end{array}$ & $\begin{array}{l}\text { Patient specific protocols for physical } \\
\text { therapy and rehabilitation } \\
\text { Weaning protocols } \\
\text { Noninvasive ventilation for weaning } \\
\text { Tracheostomy decannulation protocols } \\
\text { Prevention and treatment of nosocomial } \\
\text { infections } \\
\text { Nutrition and glucose control } \\
\text { Inflammation and immune exhaustion }\end{array}$ \\
\hline $\begin{array}{l}\text { Communication and } \\
\text { end-of-life care }\end{array}$ & $\begin{array}{l}\text { Scheduled standardized family meetings } \\
\text { Outcome prediction and prognosis } \\
\text { Palliative care consultation or protocols } \\
\text { Decision aids }\end{array}$ \\
\hline Care settings & $\begin{array}{l}\text { Comparative effectiveness studies } \\
\text { Cost-effectiveness studies }\end{array}$ \\
\hline
\end{tabular}

likelihood of CCI. Perhaps one of the most important is normal tidal volume in acute lung injury, which improves mortality in addition to ventilator days and ICU stay. ${ }^{1}$ Other practices associated with reduced mortality and ICU stay include daily interruption of sedation combined with spontaneous breathing trials. ${ }^{2}$ Early goal directed therapy improves mortality and reduces ICU stay in patients with severe sepsis; multicenter trials are underway to confirm these findings. ${ }^{3}$ Conservative fluid management in acute lung injury reduces ventilator days, ${ }^{4}$ and prevention of ventilator-associated pneumonia and catheter associated line infections may also be associated with improved outcomes. ${ }^{5}$ The difficulty lies with translation of all of these principles into daily practice. Numerous studies indicate that, despite the evidence base, clinicians are slow to put them into practice.

There are evidence-based approaches to disseminating knowledge and changing behavior of clinicians, and implementation research is needed in critical care to improve practice. A recent study by Scales and colleagues ${ }^{6}$ provides an excellent example of how multiple approaches to dissemination can be combined in an effective intervention, and their innovative study design has advanced research in the field of implementation. Investigators conducted a cluster-randomized pragmatic trial to assess the benefit of a quality improvement intervention at 15 community hospitals in Ontario, Canada. ICUs were randomized into 2 groups, each group receiving interventions to improve one of 2 care practices while serving as a control for the other group receiving the other care prac- tice intervention. The trial consisted of 3 phases, for a total of 6 interventions. The interventions consisted of educational outreach using telecommunications, audit and feedback, and reminders. During each phase they determined the difference in the change in proportion of patients receiving each targeted care practice in the intervention ICUs, compared with the same practice in the control ICUs. The primary outcome of the trial was the summary of odds ratios for all practices, calculated by pooling the ratios of odd ratios for individual practices. Patients in ICUs receiving active intervention were more likely to receive the targeted care practice than those in contemporaneous control ICUs (summary ratio of odd ratios $2.79,95 \%$ CI, $1.00-7.74, P=.05)$. Greatest improvements were noted in processes to prevent catheter related bloodstream infections and assessment of decubitus ulcer risk. This study design is complex, but the advantages are that the infrastructure developed to improve one process of care can be used to introduce and study multiple additional interventions with fewer numbers of institutions and patients.

Other practices that have the potential to reduce the incidence of CCI are currently under study. One of the most important is the concept of early versus late tracheostomy. Early clinical trials indicated that early tracheostomy for patients at risk for prolonged ventilation results in fewer ventilator days and decreased ICU stay, despite no clear benefit in mortality. ${ }^{7}$ Recent multicenter randomized trials have confirmed that there is no mortality benefit for early tracheostomy, but results for ventilator days and ICU stay are conflicting. ${ }^{8}$ They also indicate that early prediction of prolonged mechanical ventilation is challenging, so a large number of patients in the early tracheostomy groups received tracheostomies but did not need them. A similar large multicenter randomized controlled trial has not been performed in North America. However, such a clinical trial should await further research on developing effective systems to predict prolonged mechanical ventilation. Another informative topic would be qualitative studies asking patients how many extra days of mechanical ventilation they would be willing to undergo to avoid a tracheostomy, assuming there is no survival benefit, as recent trials indicate.

Another area of future research to help reduce ICU stay should be better approaches to the prevention or treatment of delirium. It is evident that benzodiazepines, especially long-acting types, are associated with increased risk of delirium and more ventilator days. ${ }^{9,10}$ However, more comparisons between shorter acting sedatives, including propofol and dexmetatomidine, are needed, and further research into management of comfort without sedatives is 
necessary. A clinical trial is underway to study the efficacy of antipsychotics for the treatment of delirium, and drug development research should focus on finding alternative agents.

The great promise of early mobility in the acute ICU should be confirmed in additional multicenter randomized trials. ${ }^{11}$ Special attention should be given to distinguishing the impact of better sedative interruption/elimination from the benefits of exercise. Early mobility is also a topic for implementation research and cost-effectiveness studies, which would aid ICU directors in securing the necessary resources.

\section{Patient Management}

Many of the practices that are targets for implementation studies and quality improvement efforts in acute ICUs to reduce the incidence of $\mathrm{CCI}$ are targets for refinement and study in settings that focus on the care of CCI patients. ${ }^{12}$ Aggressive rehabilitation has been an important cornerstone to the special care unit/long-term acute care hospitals (LTAC) model, and many are already skilled in mobilizing ventilated patients. However, this may not be uniform, so dissemination efforts should be undertaken, with research conducted to confirm effectiveness. It is possible that the primary reason for neuromuscular weakness is systemic inflammation in the acute phase of critical illness and that active physical rehabilitation during the CCI phase does not reverse that injury or significantly improve outcomes. In fact, overly aggressive physical therapy could interfere with weaning and extend the acute care stay. More likely, there may be subgroups of CCI patients who benefit from physical therapy more than others, and identification of those subgroups could help direct resource use.

Because of tracheostomies and time away from their acute event or injuries, CCI patients do not usually need as much sedating medication. However, pain, anxiety, and depression are still common, ${ }^{13-15}$ and research is needed on effective treatment regimens that are targeted to objective assessments of need. There has been some interest in the past in stimulant medications to improve motivation of depressed patients during weaning, but this should be studied for efficacy and potential complications. Similarly, the prevention of delirium can be modeled on effective approaches in the acute ICU and hospital ward, but unique needs of CCI patients may warrant specialized approaches. The concept of cognitive rehabilitation during CCI is particularly intriguing.

Specialized care units and LTACs specialize in weaning the CCI patient, so it is striking that there are few comparisons of weaning approaches. ${ }^{16}$ Many centers have substantial experience with established protocols, so efficacy studies require only organization and funding. Proprietary concerns are almost certainly overrated, as every center would benefit from what can be learned from clinical trials. Aggressive weaning approaches based on daily spontaneous breathing trials currently favored in the acute ICU patient may be detrimental to the debilitated CCI patient. However, the degree and length of work/rest cycles of more deliberate weaning approaches are intuitive at best. Randomized comparisons of clinical protocols would be useful. The best clinical protocols could then be compared to newer closed-loop ventilation modes that may be more sensitive to the tenuous balance of strength and load in the CCI patient. The role of noninvasive ventilation as an adjunct to weaning protocols beyond patients with COPD is also an important topic.

Nosocomial infections are the most common immediate cause of death in CCI patients, so care would certainly benefit from research into prevention and treatment of these complications. ${ }^{17}$ While ventilator-associated pneumonia is surely an issue, catheter related blood stream infections and urinary tract infections are more common. A checklist approach to central line insertion may be helpful in the acute setting, and similar approaches should be studied for insertion of peripherally inserted central catheter lines and tunneled catheters in the CCI patient. Research is needed on whether these lines should be changed periodically and what type of infections can be effectively treated through the lines. Similar protocols regarding indication and timing of changes for indwelling bladder catheters should also be studied.

Tracheobronchitis is common in CCI patients, but its clinical importance, including risk of progression to ventilator-associated pneumonia, is unclear. The indications for and duration of therapy are worthy research questions. Standard definitions and enrollment criteria should first be established. New formulations of inhaled antibiotics may present useful treatment options, but efficacy should be established with assessments of complications such as antibiotic resistance in individual patients and in hospital flora. The high prevalence of multiple-drug-resistant organisms in patients admitted to LTACs warrants research into the cost-effectiveness of routine surveillance and mandatory isolation on admission until patient specific resistance patterns are known. The value of rotating hospital wide antibiotic regimens has not been established in acute hospital ICUs, but this question should be studied in the high risk LTAC setting.

The literature on proper nutrition in the acutely ill patient is complex, and although research in nutrition for the CCI patient is most likely to be just as complicated, it is essential. ${ }^{18}$ Along with reducing inflammation and reversing the catabolic state, nutrition coupled with exercise is likely to be the primary avenue to restoring muscle 
function. Patient specific approaches that account for where a given patient is on the continuum of acute to chronic critical illness are needed. Parallel research on factors associated with nutrition, such as ideal glucose control, is also important. Ideal regimens may need to be balanced by resource issues. For example, long-term insulin infusions are impractical and possibly harmful, so implementation of proven subcutaneous insulin regimens should be studied for outcomes and complications. The role and timing of nutritional supplements such as vitamin D are topics of interest, and the role of anabolic steroids is worthy of further study, since initial studies were inconclusive.

Strong anti-inflammatory agents such as steroids, and other agents such as non-steroidal anti-inflammatory drugs are not clearly effective in acute lung injury or sepsis, but observational data suggest that statin medications are associated with better survival in patients with sepsis. ${ }^{19,20}$ Randomized trials are underway to assess the efficacy of statins to improve survival in sepsis and acute lung injury. Regardless of the outcome of those studies (unless there is clear harm), the efficacy of statin administration through the phase of CCI should be studied, because current studies of statin use in the acute phase mostly employ limited courses of therapy.

Recent data have advanced the concept that acute critical illness is associated with immune exhaustion. ${ }^{21}$ These findings strengthen hypotheses that immune enhancement or supplementation could improve outcomes from acute and chronic critical illness. More basic science is needed to identify potentially successful interventions, but this represents a very important shift in focus on how patient outcome can be impacted.

\section{Communication and End-of-Life Care}

Studies involving surrogate decision-makers for patients with CCI have indicated that they receive insufficient information regarding prognosis and options for care. ${ }^{22-24}$ This is especially true for long-term survival and functional outcomes. Further research is needed regarding whether the gaps in understanding relate to deficiencies in communication on the part of clinicians or to difficulties in understanding by the surrogates. Regardless, there appears to be ample need for effective interventions to improve communication between clinicians and patients and their surrogates. ${ }^{25}$ Improvements in existing models of outcome prediction are necessary, especially for predicting functional outcomes in long-term survivors. A multicenter randomized trial is underway to determine if additional support and information provided by clinicians trained in palliative care can improve patient- and family- centered outcomes. A pilot study will soon be published suggesting that such outcomes can be improved with the use of a decision support tool targeted to individual patients requiring prolonged mechanical ventilation. ${ }^{26}$ This should be confirmed in a larger multicenter clinical trial.

Evidence has been increasing on the benefit of palliative care medicine for patients with advanced illnesses who are at the end of life. ${ }^{27,28}$ Importantly, palliative care can improve patient-centered outcomes, even when patients are not at the end of life. There is clearly a role for palliative care specialists and protocols in any care setting for patients with CCI, considering their high symptom burden and high mortality. It will be quite some time before there are enough palliative care specialists available to consult on every CCI patient, much less all of the other hospitalized patients and out-patients with symptomatic advanced conditions. In the meantime, palliative care specialists should be recruited to help design protocols and educational interventions for bedside nurses and clinicians that can be studied for impact on practice patterns and patient outcomes.

\section{Care Settings}

Ideal care settings for CCI patients will remain a topic of interest due to their unique care needs and the high costs associated with their management. The rehabilitation model of care for CCI patients is well accepted, and some evidence exists supporting this model in different settings (LTACs and other specialized weaning centers, dedicated units in acute hospitals, dedicated teams in acute ICUs). Current observational studies are small, and results of population based studies using administrative data sets conflict with prospective observational studies. ${ }^{29,30}$ Large multicenter randomized trials would be ideal to compare costs and outcomes in different settings, but this would be logistically very difficult unless major payers such as the Centers for Medicare and Medicaid Services are committed to such a project. In the meantime, additional multicenter observational studies (using large data sets and prospective cohorts) with advanced modeling to adjust for patient selection should be conducted to add to the growing literature on this topic.

\section{Summary}

Many of the existing research programs in the acute care setting have potential to impact the field of CCI by reducing the incidence of $\mathrm{CCI}$ or improving the condition of patients as they evolve into the chronic phase of critical illness. Research into improving dissemination and implementation of evidence-based practices that reduce the degree of organ failure or prolonged brain or muscle dysfunction could reduce the downstream burden of CCI. 


\section{Research Needs and Strategies for Chronic CRitical Illness}

There are numerous potential interventions that could improve the management and outcome of patients who have developed CCI. Concentration of patients within weaning facilities presents excellent opportunities to compare protocols for weaning, nutrition and glucose control, infection control, and palliation of symptoms. Basic science research should advance to better characterize the biological transformations in immune function, as well as muscle, brain, and lung function. Communication of prognosis and establishment of patient goals of care can be improved by exploring different approaches to presenting and receiving information during interactions between clinicians and surrogate decision-makers. Finally, health services research should continue attempts to provide a logical framework for efficient systems to manage the increasing patient needs and resources presented by CCI.

\section{REFERENCES}

1. The Acute Respiratory Distress Syndrome Network. Ventilation with lower tidal volumes as compared with traditional tidal volumes for acute lung injury and the acute respiratory distress syndrome. N Engl J Med 2000;342(18):1301-1308.

2. Girard TD, Kress JP, Fuchs BD, Thomason JW, Schweickert WD, Pun BT, et al. Efficacy and safety of a paired sedation and ventilator weaning protocol for mechanically ventilated patients in intensive care (Awakening and Breathing Controlled trial): a randomised controlled trial. Lancet 2008;371(9607):126-134.

3. Rivers E, Nguyen B, Havstad S, Ressler J, Muzzin A, Knoblich B, et al. Early goal-directed therapy in the treatment of severe sepsis and septic shock. N Engl J Med 2001;345(19):1368-1377.

4. The National Heart, Lung, and Blood Institute ARDS Clinical Trials Network. Comparison of two fluid-management strategies in acute lung injury. N Engl J Med 2006;354(24):2564-2575.

5. Pronovost P, Needham D, Berenholtz S, Berenholtz S, Sinopoli D, $\mathrm{Chu} \mathrm{H}$, et al. An intervention to decrease catheter-related bloodstream infections in the ICU. N Engl J Med 2006;355(26):2725-2732.

6. Scales DC, Dainty K, Hales B, Pinto R, Fowler RA, Adhikari NK, Zwarenstein M. A multifaceted intervention for quality improvement in a network of intensive care units: a cluster randomized trial. JAMA 2011;305(4):363-372.

7. Griffiths J, Barber VS, Morgan L, Young JD. Systematic review and meta-analysis of studies of the timing of tracheostomy in adult patients undergoing artificial ventilation. BMJ 2005;330(7502): 1243.

8. Terragni PP, Antonelli M, Fumagalli R, et al. Early vs late tracheotomy for prevention of pneumonia in mechanically ventilated adult ICU patients: a randomized controlled trial. JAMA 2010;303(15): 1483-1489.

9. Pandharipande P, Shintani A, Peterson J, Pun BT, Wilkinson GR, Dittus RS, et al. Lorazepam is an independent risk factor for transitioning to delirium in intensive care unit patients. Anesthesiology 2006;104(1):21-26.

10. Riker RR, Shehabi Y, Bokesch PM, Ceraso D, Wisemandle W, Koura $\mathrm{F}$, et al. Dexmedetomidine vs midazolam for sedation of critically ill patients: a randomized trial. JAMA 2009;(5):489-499.

11. Schweickert WD, Pohlman MC, Pohlman AS, Nigos C, Pawlik AJ, Esbrook CL, et al. Early physical and occupational therapy in mechanically ventilated, critically ill patients: a randomised controlled trial. Lancet 2009;373(9678):1874-1882.
12. Needham DM, Davidson J, Cohen H, Hopkins RO, Weinert C, Wunsch $\mathrm{H}$, et al. Improving long-term outcomes after discharge from intensive care unit: report from a stakeholders' conference. Crit Care Med 2012;40(2):502-509.

13. Nelson JE, Meier DE, Litke A, Natale DA, Siegel RE, Morrison RS. The symptom burden of chronic critical illness. Crit Care Med 2004; 32(7):1527-1534.

14. Jubran A, Lawm G, Kelly J, Duffner LA, Gungor G, Collins EG, et al. Depressive disorders during weaning from prolonged mechanical ventilation. Intensive Care Med 2010;36(5):828-835.

15. Jubran A, Lawm G, Duffner LA, Collins EG, Lanuza DM, Hoffman LA, Tobin MJ. Post-traumatic stress disorder after weaning from prolonged mechanical ventilation. Intensive Care Med 2010;36(12): 2030-2037.

16. Scheinhorn DJ, Chao DC, Stearn-Hassenpflug M, Wallace WA. Outcomes in post-ICU mechanical ventilation: a therapist-implemented weaning protocol. Chest 2001;119(1):236-242.

17. Kalb TH, Lorin S. Infection in the chronically critically ill: unique risk profile in a newly defined population. Crit Care Clin 2002;18(3): 529-552.

18. Hollander JM, Mechanick JI. Nutrition support and the chronic critical illness syndrome. Nutr Clin Pract 2006;21(6):587-604.

19. Janda S, Young A, Fitzgerald JM, Etminan M, Swiston J. The effect of statins on mortality from severe infections and sepsis: a systematic review and meta-analysis. J Crit Care 2010;25(4):656, e657-e622.

20. Kor DJ, Iscimen R, Yilmaz M, Brown MJ, Brown DR, Gajic O. Statin administration did not influence the progression of lung injury or associated organ failures in a cohort of patients with acute lung injury. Intensive Care Med 2009;35(6):1039-1046.

21. Boomer JS, To K, Chang KC, Takasu O, Osborne DF, Walton AH, et al. Immunosuppression in patients who die of sepsis and multiple organ failure. JAMA 2011;306(23):2594-2605.

22. Nelson JE, Kinjo K, Meier DE, Ahmad K, Morrison RS. When critical illness becomes chronic: informational needs of patients and families. J Crit Care 2005;20(1):79-89.

23. Nelson JE, Mercado AF, Camhi SL, Tandon N, Wallenstein S, August GI, Morrison RS. Communication about chronic critical illness. Arch Intern Med 2007;167(22):2509-2515.

24. Cox CE, Martinu T, Sathy SJ, Clay AS, Chia J, Gray AL, et al. Expectations and outcomes of prolonged mechanical ventilation. Crit Care Med 2009;37(11):2888-2894.

25. Scheunemann LP, McDevitt M, Carson SS, Hanson LC. Randomized, controlled trials of interventions to improve communication in intensive care: a systematic review. Chest 2011;139(3):543-554.

26. Cox CE, Lewis CL, Hanson LC, Hough CL, Kahn JM, White DB, et al. Development and pilot testing of a decision aid for surrogates of patients with prolonged mechanical ventilation. Crit Care Med 2012; in press.

27. Norton SA, Hogan LA, Holloway RG, Temkin-Greener H, Buckley MJ, Quill TE. Proactive palliative care in the medical intensive care unit: effects on length of stay for selected high-risk patients. Crit Care Med 2007;35(6):1530-1535.

28. Temel JS, Greer JA, Muzikansky A, Gallagher ER, Admane S, Jackson VA, et al. Early palliative care for patients with metastatic nonsmall-cell lung cancer. N Engl J Med 2010;363(8):733-742.

29. Kandilov AMG, Dalton K. Utilization and payment effects of Medicare referrals to long-term care hospitals (LTCHs). Final Report June 2011. http://www.rti.org/reports/cms/HHSM-500-2006-00008I/PDF/ CMS_LTCH_Referral_Effects.pdf. Accessed March 30, 2012.

30. Votto JJ, Scalise PJ, Barton RW, Vogel CA. An analysis of clinical outcomes and costs of a long term acute care hospital. J Med Econ 2011;14(2):141-146. 


\section{Discussion}

MacIntyre: That was a nice wrap-up of where we need to go. There's a lot to do, isn't there? If I may ask my 3 industry colleagues here, is it feasible that you could work together to do some of these large scale trials?

Bertuola:* We definitely would like to get involved. We'd have to decide what it is we want to look at.

MacIntyre: This always involves money, but is the corporate culture in your institutions such that an investment into looking at best practices would be viewed as something worthwhile?

Muldoon: $\dagger$ We are certainly open to talking about it, but in our previous attempts to do clinical research we were not able to put together the magic 5 "P"s: physicians, patients, place, protocols, and pesos. But that doesn't mean it can't be done! As Shannon said, there would have to be a real coming together that we haven't had before.

Snyder: $\$$ I would like to think that it's hopeful. I think all the companies talk about it. We talk about it at our industry society meetings, that it needs to be done. Maybe we can start looking at that and see if we can cross some of those barriers. We have already started to work with some large university settings to try and get research on the board so that we have the expertise from the university researchers to really make the projects credible and valid. I think this is a great springboard for us to continue to work on that.

\footnotetext{
* Lorraine Bertuola RRT, Genesis Healthcare, Kennett Square, Pennsylvania.

$\uparrow$ Sean R Muldoon MD MPH, Kindred Healthcare, Hospital Division, Louisville, Kentucky. $\ddagger$ Lisa Snyder MD MPH, Select Medical, Mechanicsburg, Pennsylvania.
}

MacIntyre: Shannon, you brought up trying to get propensity scores as a poor man's surrogate for randomized controlled trials. It seems that there's a long track record that this is often not a good idea. The ARDS Network did a study on pulmonary artery catheters versus central venous lines because Al Connor's propensity scores implied that we were killing people with pulmonary artery catheters. A lot of people argue with that, saying that propensity scores can lead you down the wrong path. And, sure enough, the study found that pulmonary catheters didn't kill. ${ }^{1}$ They may not help, but at least they won't kill you. Is there a valid role for propensity score substitution for randomized controlled trials?

1. National Heart, Lung, and Blood Institute Acute Respiratory Distress Clinical Trials Network; Wheeler AP, Bernard GR, Thompson BT, Schoenfeld D, Wiedemann HP, deBoisblanc B, et al. Pulmonary-artery versus central venous catheter to guide treatment of acute lung injury. N Engl J Med 2006;354(21):2213-2224.

Carson: Yes, you're going to see more of it because of the renewed emphasis on comparative effectiveness research in everything. The patientcentered outcomes research initiative is part of the health care plan that is going to fund comparative effectiveness research, and the reality is that most effectiveness research can only be done in observational settings. Most people will say that observation studies with propensity matching and other forms of matching is hypothesis-generating; you can't evaluate interventions effectively with it: you need a randomized controlled model for that. But when you talk about effectiveness, a randomized controlled model is not practical in many settings. You have to look carefully at what goes into the propensity score and is it enough?

My worry from the beginning on the propensity scoring approach was, in both instances, you just can't measure the sense of "does this patient need prolonged critical care?" This can only be done on the spot, prospectively, and that would be a very difficult type of study to do. In some settings propensity scoring matching is very good, and in others challenging and difficult. This one I think falls into challenging and difficult.

Snyder: As was highlighted in the very first talk, we talked about definitions, which is a great way to start. And as we continue down the education continuum of CCI, it's my hope that we will move towards a clinical data set. A clinical definition as opposed to an administrative data set, for the reasons that you've highlighted, as far as what the limitations are. I liked the idea of the tracheostomy being a trigger point, but that's for the patients who are CCI on a ventilator.

I think we still need and are still searching for the trigger to call a CCI patient when they're not tracheostomized and not on a ventilator, and we know that those patients definitely exist. It's another challenge we have on the research side; the clinical side is to figure out what that trigger may be.

Our definitions are going to be important as we try to do research. In the study from the RTI [Research Triangle Institute] that you highlighted, ${ }^{1}$ what was the end point in the difference in days? What stopped the day calculation? Was it home? Was it to a skilled nursing facility or another postacute care facility?

\section{Kandilov AMG, Dalton K. Utilization and payment effects of Medicare referrals to long-term care hospitals (LTCHs). Final Re- port June 2011. http://www.rti.org/reports/ cms/HHSM-500-2006-00008I/PDF/ CMS_LTCH_Referral_Effects.pdf. Accessed March 30, 2012.}

Carson: I believe it was discharge from acute care, meaning either shortterm or LTAC hospital was the end point for measurement costs. You raise an important question: if they're discharged from short-term acute care, is 
the patient going to a nursing home and receiving institutional services there? I believe there was no difference between the groups in nursing home days, or that it was small, by 3 days or so. I think they thought of that and looked at it.

White: Shannon, there's no measure of function of a patient at the time of discharge in the RTI database, because it is an administrative database and none of that information is available. We have a small research center in the LTAC where I work, and it really takes a champion and somebody in that institution to drive it forward and to have the weekly meetings and twist the arms of the hospital into providing the money for a research assistant. I think it would be great if we could form a CCI network, like the ARDS Network, and link all of us together to define what data we want to collect and what questions we want to ask.

Carson: I agree, and such a network does not have to be huge. The key is commitment and dedication of the cen- ters involved. And then, once you're up and running, you can apply for federal funding the same way an academic hospital does. In fact, the way things are going, with so much more focus on effectiveness and real-world care, I think they'd be excited to get a dedicated academic-based network out in the real-world setting. You introduced yourself as being from an academic LTAC: that's what we want to hear.

Nelson: Universities are not going to be able to do this research on their own, and LTACs can't do it alone. Most CCI patients are being cared for in LTACs, so it makes sense to have a collaborative effort between the academic research community and the LTAC community. Ideally, LTACs would contribute some resources, form an alliance with academic researchers, and make a commitment to a research network that could seek extramural funding.

Muldoon: That's a much more doable first step. On the CCI front, do we separate from or join with the
Society of Critical Care Medicine, which is working and publishing on something called post-intensive-care syndrome? Are these likely to come together or stay as separate entities, from both a common language perspective and research objective?

Carson: If there are opportunities to learn and benefit from cooperation with others on what's pretty much a common cause, getting in line with that effort could be beneficial. What they describe in the post-acute-care syndrome is a lot of what we're describing here, but here there's more severity: the patient is still dependent on a life support system of some sort, and even if they get off it, they still need considerable specialized care.

I was struck by the data from the Pittsburgh cohort, where the 3-month physical limitations of patients with short-term ventilation were still pretty high. There are a lot of common themes here and probably a lot that can be beneficial for all patient groups. 